\title{
A 3D Nonlinear Poisson Solver
}

\author{
GYULA VESZELY \\ Department of Electromagnetic Theory, Budapest Technical University, 1521 Budapest, Hungary
}

\begin{abstract}
The Poisson equation is solved in a rectangular prism of semiconductor with the boundary conditions commonly used in semiconductor device modeling. There is a planar heterojunction inside the prism. The finite difference formulation leading to a matrix of seven diagonals is used. The 3D version of the Stone's method is applied for the iterative solution of the matrix equation. The nonlinear dependence of the carrier concentration on the electrostatic potential is taken into account. The heterojunction is modeled by a potential jump. The advantages and limits of the method is presented.
\end{abstract}

Keywords: Poisson's equation, semiconductor device modeling, Stone's method, heterojunction

\section{PROBLEM STATEMENT}

The solution of the nonlinear Poisson's equation is a fundamental task in the modeling of semiconductor devices [1]. The problem to be examined was formulated in Ref. [2]. Figure 1 shows a possible realization in a rectangular geometry. The prism is combined from two different semiconductors contacting in a planar heterojunction. On the lateral faces and on the base zero Neumann boundary condition is supposed. On the upper surface on the rectangular spots (these are the exposed surfaces) zero Neumann boundary condition, on the metallized remaining part (this is the gate) Dirichlet condition is prescribed respectively. Chen and Porod [2] solved the problem by a coupled finite element/boundary element method. The drawback of this method is that it demands a matrix bandwidth reduction and a Newton iteration. The purpose of the present paper to solve the problem by the finite difference method combined with a 3D version of the Stone's [3] method.

\section{NUMERICAL TREATMENT}

A standard finite difference discretization is applied with variable grid distances. The boundary conditions can be taken into account in the usual manner. At the zero-Neumann boundaries mirror potentials were used, while at the $i^{\text {th }}$ surface point where the potential is prescribed to be $\Phi_{\text {given }}$ the equation $\Phi_{i}=\Phi_{\text {given }}$ is valid, i.e., in the $i^{\text {th }}$ row all of the matrix elements are zero except the main diagonal element, which equal to unity and at the right-hand side of the equation in the column vector of the excitation the $i^{\text {th }}$ element is $\Phi_{\text {given }}$. Continuously numbering the grid points a sparse matrix with seven diagonals is resulted. 

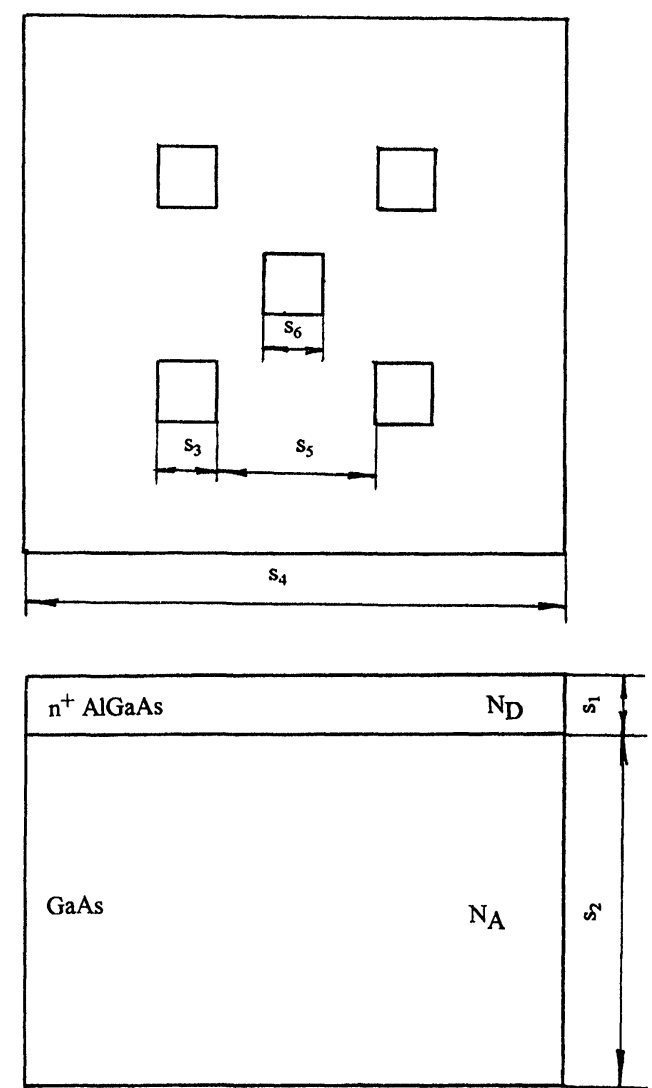

FIGURE 1 The structure to be analyzed. The upper part is a top view, while the lower part is a side-view. The small squares on the top view are the exposed surfaces.

The heterojunction is characterised by a potential jump [4]. This condition must be built into the sytem of equations so that no excess matrix elements to be appeared. We enforce the potential jump by a double layer. The connection between the moment of the doble layer and the given potential jump is

$$
\sigma \Delta n=\varepsilon \Delta \Phi
$$

where $\sigma$ is the surface charge density, $\Delta n$ is the distance between the positive and negative layer, $\varepsilon$ is the permittivity and $\Delta \Phi$ is the potential jump characterizing the heterojunction (Fig. 2). Using the Gauss-theorem f.e. for the upper volume element in Figure 2 the field strengthes can be

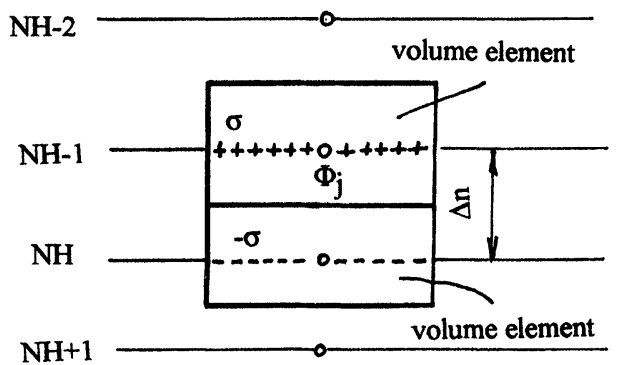

FIGURE 2 The potential jump is modeled by a double layer The Gauss-theorem is applied to the volume element.

calculated as the potential differences divided by the appropriate distances. So $\Phi_{j}$ and all of the six neighbouring potentials appear in the $j^{\text {th }}$ equation as in an "ordinary" point (without surface charge). The given charge enclosed by the volume will be an element of the right-hand side column vector. The distance $\Delta n$ is choosed $0,01^{\text {th }}$ part of the neighbouring (to the surface perpendicular) grid distances.

The treatment of the potential dependent carrier density is according to [5]. This is fitted itself naturally to the iterative procedure of Stone.

\section{CONVERGENCE PROPERTIES}

The numerical results are calculated under the following data (see Fig. 1): the depth of the heavily doped semiconductor $s_{1}=10 \mathrm{~nm}$, the depth of the substrate $s_{2}=110 \mathrm{~nm}$, the side length of the squares of the satellite excited surfaces $s_{3}=10 \mathrm{~nm}$, the side length of the rectangular prism $s_{4}=120 \mathrm{~nm}$, the distance between two satellite excited surface $s_{5}=40 \mathrm{~nm}$, the side length of the square of the central excited surface $s_{6}=10 \mathrm{~nm}$, the donor density of the heavily doped part $N_{\mathrm{D}}=10^{18} \mathrm{~cm}^{-3}$, the acceptor density of the substrate $N_{\mathrm{A}}=10^{15} \mathrm{~cm}^{-3}$, the voltage characterizing the Schottky contact is $0,8 \mathrm{~V}$, the potential jump at the heterojunction is $0,3 \mathrm{~V}$. The gate voltage is $-1 \mathrm{~V}$. 
When the number of unknowns exceeds a critical level the convergence speed degrades drastically (Fig. 3). 35 min CPU time is required for the 1700 iteration on a HP $9000715 / 80$ workstation. As it is known the $\alpha$ iteration parameter of the Stone's method is an important but theoretically not well established quantity. Most authors using the method have their own recipe for choosing the iteration parameter. We used the following formula proposed by Stone [3]

$$
\alpha=1-b^{e}
$$

where $e$ varied cyclically with the values 0,$6 ; 0,4$; 0,$2 ; 0,8 ; 0,2 ; 1$. $b$ generally equal to $10^{-3}$ but in the case of the curve a) in Figure 3 we had to change it to $10^{-2}$ at the iteration number 100 otherwise divergence occured.

\section{RESULTS}

The electron potential energy was calculated at the substrate side of the heterojunction Figure 4 shows a contour plot, while Figure 5 a surface plot of the

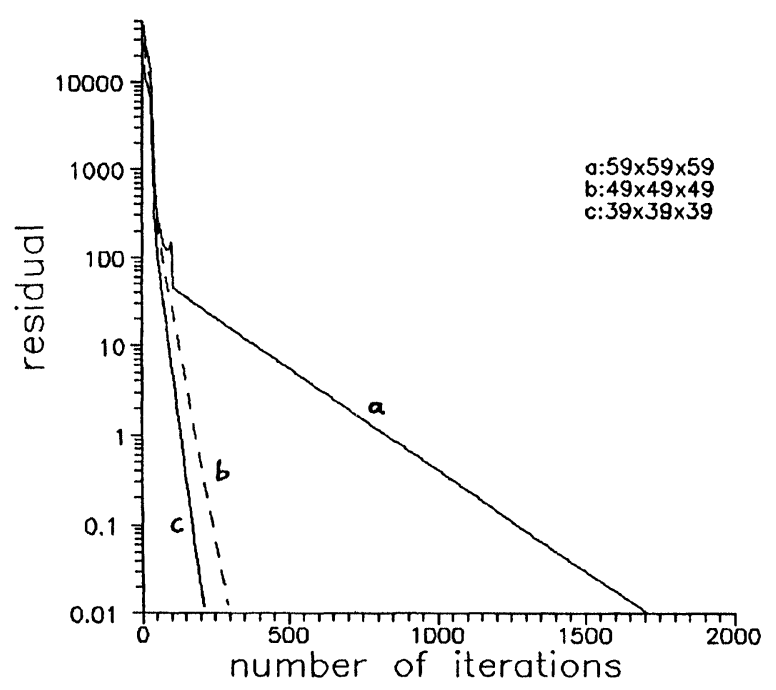

FIGURE 3 The residual versus the iteration number. In the inlet the numbers of grid point can be seen.

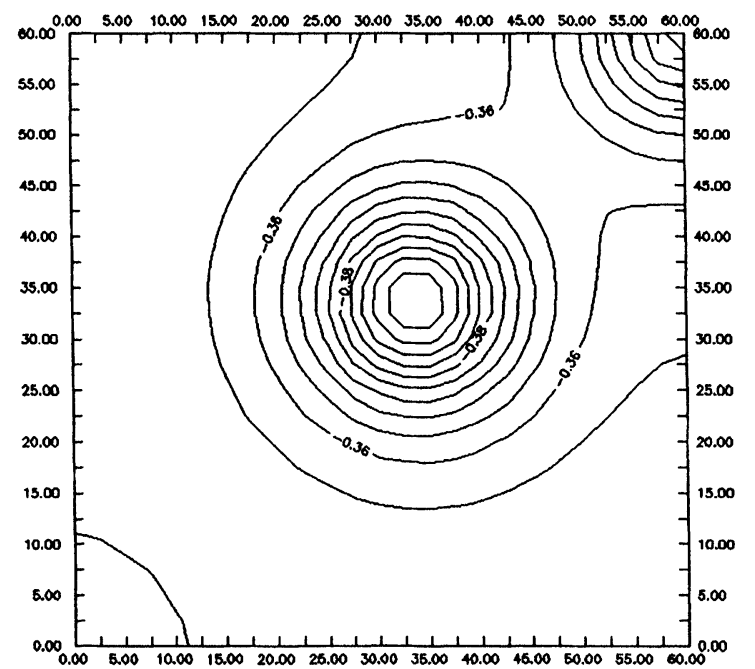

FIGURE 4 Contour plot of the electron potential energy. One satellite dot and a quarter of the central dot can be seen.

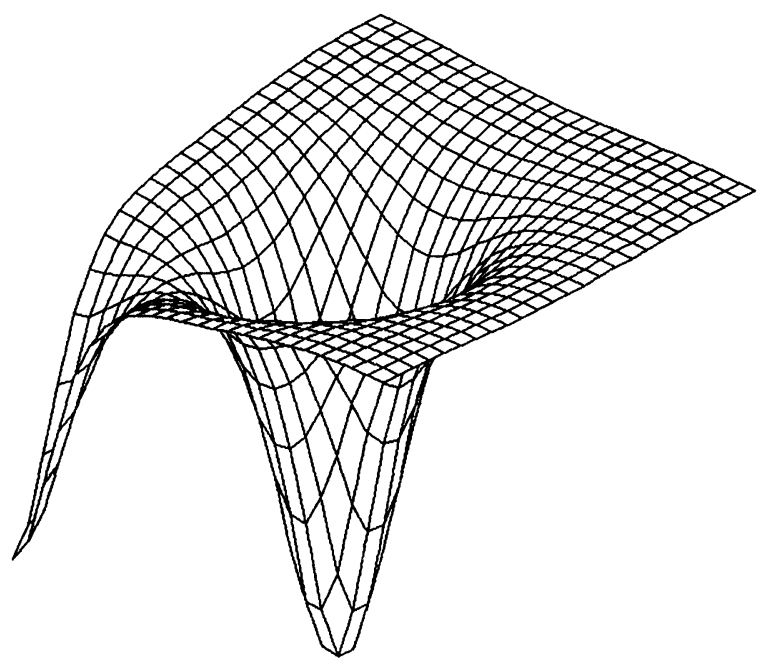

FIGURE 5 Surface plot of the electron potential energy. One satellite dot and a quarter of the central dot can be seen.

energy. The diagrams presented are calculated under the grid dimension $59 \times 59 \times 59 \approx 205000$. In the vertical direction in the heavily doped part 50 grid points was applied uniformly and only 9 one in the substrate, the distances of the latter are increasing as a geometrical series. Using the symmetry only one quarter of the arrangement was calculated. 
By the method the ratio of the depth of the central and satellite valleys and the absolute depth of the valleys can be calculated. These parameters, which are important in the charging and recharging process can be tailored by the proper choice of the geometry and the gate voltage.

\section{CONCLUSIONS}

The electrostatic problem of a semiconductor quantum device with exposed surface was solved by the finite difference/Stone's method.

The advantages of the method are: simple programing, low storage requirement, the iteration demanded by the nonlinearity can be naturally built into the iteration type solution.

The drawbacks are: after a limit the convergence becomes rather slow, there is no possibility to the local refinement of the grid.

The application of a more advanced iterative solver [6] is under development.

\section{Acknowledgments}

Valuable discussions with A. CSURGAY and W. POROD (University of Notre Dame) are gratefully acknowledged. This work has been supported by Hungarian National Science Foundation No. T-023337 and by a joint grant of NSF (National Science Foundation) and HAS (Hungarian Academy of Sciences) No. INT96-15497.

\section{References}

[1] Csurgay, A. and Simonyi, K. (1997). Physics of Electronic Information Technologies, (In Hungarian: Az információtechnika fizikai alapjai), BME Mérnöktovábbképzõ Intézet, Budapest.

[2] Chen, M. and Porod, W. "Design of Gate-Confined Quantum-Dot Structures in the Few-Electron Regime", $J$. Appl. Phys., July 1995.

[3] Stone, H. L. "Iterative Solution of Implicit Approximations of Multidimensional Partial Differential Equations", SIAM J. Numer. Anal., September 1968.

[4] Chen, M., Porod, W. and Kirkner, D. J. "Coupled Finite Element/Boundary Element Method for Semiconductor Quantum Devices with Exposed Surfaces", J. Appl. Phys., March 1994.

[5] Gummel, H. K. "A Self-Consistent Iterative Scheme for One-Dimensional Steady-State Transistor Calculations", IEEE Trans. on Electron Devices, October 1964.

[6] van der Vorst, H. A. "Bi-CGSTAB: a Fast and Smoothly Converging Variant of $\mathrm{Bi}-\mathrm{CG}$ for the Solution of Nonsymmetric Linear Systems", SIAM J. Sci. Stat. Comput., March 1992.

\section{Author Biography}

Gyula Veszely was born on December 22, 1938 in Szombathely, Hungary. He obtained the master degree in electrical engineering in 1962 from the Budapest Technical University (BTU). In 1993 he obtained the Doctor of Science degree from the Hungarian Academy of Sciences. Since 1962 he has been working at the Department of Electrical Engineering and Informatics of BTU, since 1993 he has been a Professor and head of the Chair of Electromagnetic Theory. His main research interest is in the development and application of numerical methods for computing electromagnetic fields in complicated materials and geometries. 

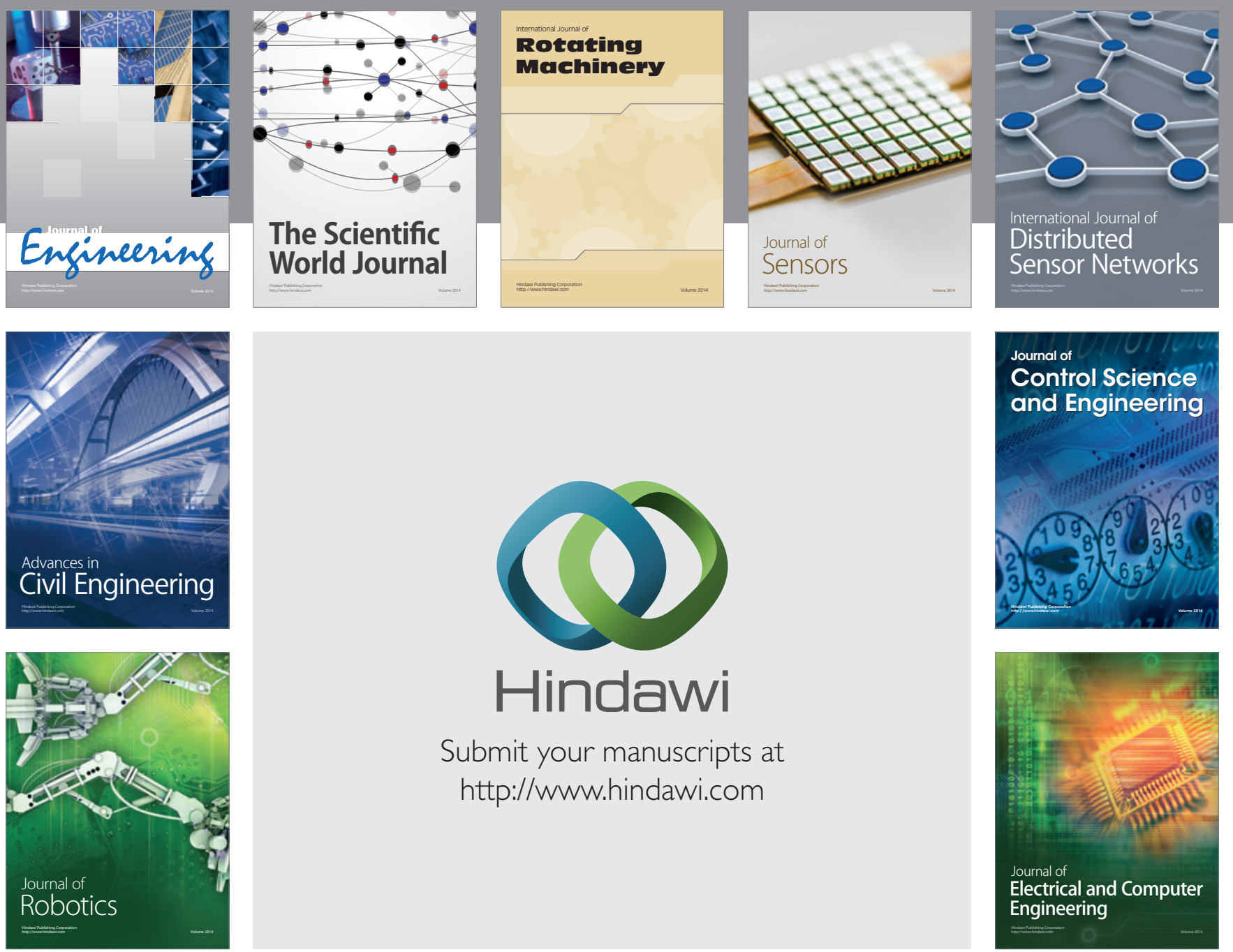

Submit your manuscripts at

http://www.hindawi.com
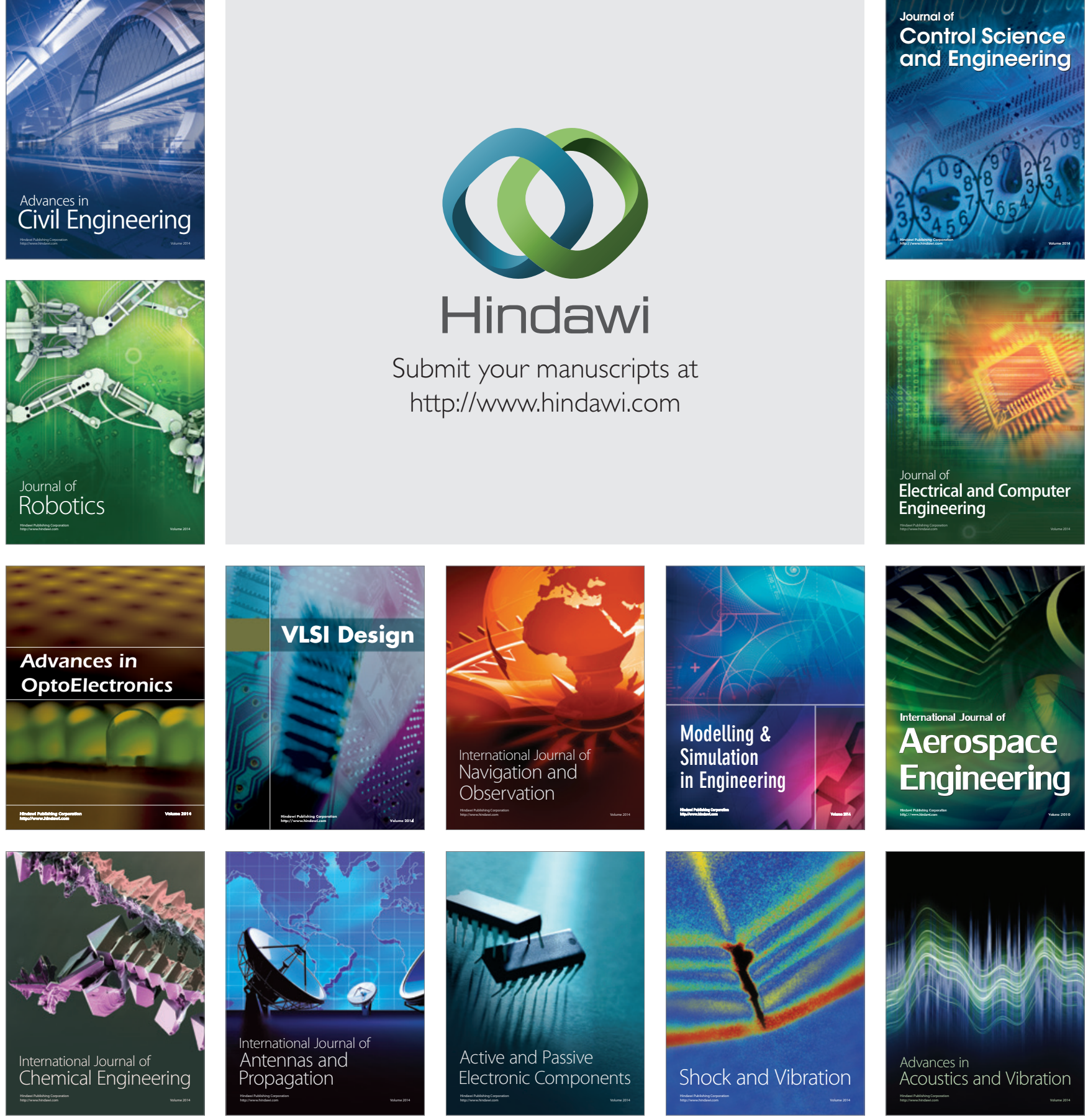